\title{
Epidermal growth factor induction of resistance to topoisomerase II toxins in human squamous carcinoma $\mathrm{A} 431$ cells
}

\author{
WING-PUI TSANG, SIU-KAI KONG and TIM-TAK KWOK \\ Department of Biochemistry, The Chinese University of Hong Kong, Hong Kong SAR, P.R. China
}

Received March 8, 2006; Accepted May 4, 2006

\begin{abstract}
Alteration of the epidermal growth factor (EGF) signaling pathway occurs frequently in human cancer cells and may subsequently affect the cell survival towards anticancer agents. To elucidate the effect of long-term EGF treatment on the chemo-sensitivity of human cancer cells, human squamous carcinoma A431 cells (AP) were incubated continuously with $50 \mathrm{ng} / \mathrm{ml}$ EGF for 30 weeks and these cells were designated as the AC cells. The long-term EGF treatment did not alter the EGFR level and the EGF-induced protein tyrosine phosphorylation pattern in the AC cells. By MTT assay, the AC cells were shown to be more resistant than the AP cells to doxorubicin, etoposide and amsacrine but not to cisplatin. Among the drug-resistant proteins, topoisomerase $\mathrm{II} \alpha$ (topoII) was downregulated in the AC cells while there was no apparent change in the levels of P-glycoprotein, MRP-1 or glutathione- S-transferase- $\pi$ as compared to the AP cells. Furthermore, knockdown of topoII by antisense topoII oligonucleotide transfection decreased the sensitivity to doxorubicin, etoposide and amsacrine in the A431 cells. Results from the present study support an idea that long-term treatment with EGF may induce drug resistance in cells through the downregulation of topoII.
\end{abstract}

\section{Introduction}

The epidermal growth factor receptor (EGFR) is a $170 \mathrm{kDa}$ transmembrane protein. Binding of the specific EGF ligand to its cognate receptor induces auto-phosphorylation on receptor tyrosine residues. A cascade of phosphorylation and protein-protein interaction is then triggered and subsequently leads to diversified cellular activities including cell proliferation, cell cycle progression, and DNA damage repair. Therefore, the EGF signaling pathway is believed to have a major influence on the drug and radiation sensitivity

Correspondence to: Dr Tim-Tak Kwok, Department of Biochemistry, The Chinese University of Hong Kong, Shatin, N.T., Hong Kong SAR, P.R. China

E-mail: kwok2020@cuhk.edu.hk

Key words: epidermal growth factor, topoisomerase, A431 cells of cells (1-5). This is supported further by the fact that many of the signaling molecules such as raf and myc are known to be the determinants for drug and radiation sensitivity in cells (6-8). Furthermore, the signaling pathways for EGF and DNA damage may have some parts or signaling molecules in common. Therefore, agents acting specifically on the EGF receptor or its downstream signaling molecules, e.g. the antibody targeting EGFR, tyrphostin, genistein, suramin, were reported not only to suppress cancer growth but also, at the time of combination with chemotherapy and radiotherapy, alter the sensitivity of cells to anticancer drugs and radiation (8-11).

Although agents acting on the signaling molecules might have benefits in cancer treatment, there are still concerns about those cells that survive the treatment. The cells that survive might demonstrate resistance to the therapeutic agents and have other cellular changes, such as an alteration in chemotherapeutic response as reported for cells surviving anticancer drug treatments. Human leukemic CCRF-CEM cells after continuous exposure to protein tyrosine kinase inhibitor genistein developed resistance to genistein and also showed cross-resistance to metabolic inhibitors (such as cytosine arabinoside, 5-fluoro-2'-deoxyuridine) and topoisomerase inhibitors (e.g. etoposide) (12). Continuous exposure of Chinese hamster fibrosarcoma DC-3 cells to suramin, another tyrosine kinase inhibitor, induced resistance to suramin and topoisomerase II inhibitors, but increased the sensitivity to the topoisomerase I inhibitor camptothecin (13). Long-term treatment with EGFR-blocking antibodies (such as C225) in vivo induced not only resistance to the therapeutic antibodies but also angiogenic potential of the cells $(14,15)$. The influence of the long-term antibody treatment on anticancer drug response has, however, not been addressed. In the present study, the influence of long-term EGF treatment on anticancer drug sensitivity of cells was investigated. The long-term EGF treatment was performed by culturing human squamous carcinoma A431 cells in the presence of $50 \mathrm{ng} / \mathrm{ml}$ EGF for 30 weeks. Results from the study suggest that long-term exposure to EGF induces topoisomerase II $\alpha$ (topoII) related drug resistance in cells.

\section{Materials and methods}

Cell lines, antibodies and drugs. The AC cells with longterm EGF treatment were established by culturing human 


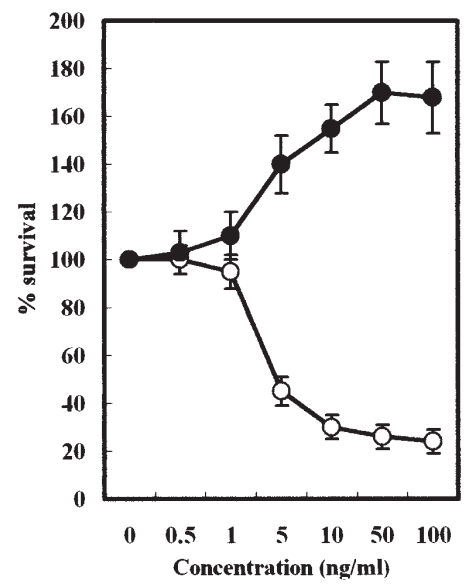

Figure 1. The response of A431 cells to EGF. Cells were grown for 2 days followed by 5 days of growth factor incubation. The dose-response curve was measured by MTT assay. -o-, AP cells; - $\bullet-$, AC cells. Each data point is the average of results from at least 4 separate experiments. Error bar, standard derivation.

squamous carcinoma A431 cells in $50 \mathrm{ng} / \mathrm{ml}$ EGF for 30 weeks. To carry out the experiment, the AC cells were first cultured for 4-5 days in a medium without the addition of EGF. Both A431 parent (AP) cells and AC cells were grown in Dulbecco's modified Eagle's medium (DMEM) supplemented with $10 \%$ fetal bovine serum (Invitrogen, USA) in a $37^{\circ} \mathrm{C}$ humidified $10 \% \mathrm{CO}_{2}$ incubator. Monoclonal antitopoisomerase II $\alpha$ and P-glycoprotein antibodies were purchased from Calbiochem (Germany). Polyclonal antiEGFR, anti-MRP1 and anti-PY-99 antibodies were purchased from Santa Cruz Biotechnology Inc., USA. Doxorubicin and cisplatin were obtained from Sigma (USA). Etoposide and amsacrine were purchased from Calbiochem.

MTT cell viability assay. Cells were seeded in a 96-well plate for 2 days and then exposed to various concentrations of drugs for $48 \mathrm{~h}$ or to EGF for 5 days. At the end of incubation, the cells were incubated with $50 \mu 1$ of $0.1 \mathrm{mg} / \mathrm{ml}$ solution of 3-[4,5-dimethylthiazol-2-yl]-2,5-diphenyltetrazolium bromide (MTT) at $37^{\circ} \mathrm{C}$ for $3 \mathrm{~h}$ and then lysed in $150 \mu 1$ of dimethyl sulfoxide (DMSO) at room temperature for $30 \mathrm{~min}$. The absorbance in each well was measured at $580 \mathrm{~nm}$ by a microplate reader.

Western blot analysis. Cells were lysed in Lammeli lysis buffer containing $1 \%$ Triton X-100 and scraped with a cell lifter. Protein $(25 \mu 1)$ was resolved in $8 \%$ SDS-PAGE and transferred to an Immobilon-P membrane (Millipore, USA). Membranes were blocked with $5 \%$ non-fat dry milk at $4{ }^{\circ} \mathrm{C}$ overnight and incubated with primary antibodies at room temperature for $2 \mathrm{~h}$. Afterwards, membranes were washed three times with $1 \mathrm{X}$ PBS in $0.1 \%$ Tween-20 and incubated with respective secondary antibodies conjugated with horseradish peroxidase at 1:10000 dilution. The signals were visualized by enhanced chemiluminescence (ECL, Amersham Life Science Inc., UK).

Transient transfection with topoll oligonucleotides. The 20base antisense oliognucleotides complementary to the coding

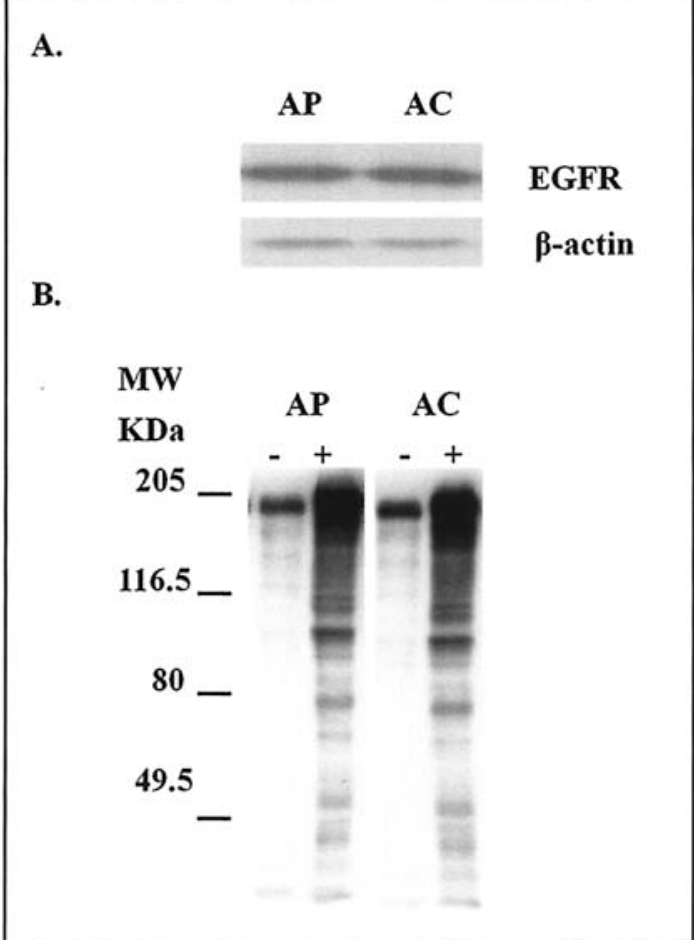

Figure 2. (A) The level of the EGF receptor and (B) the EGF induced protein tyrosine phosphorylation pattern in A431 cells as measured by Western blot analysis. Protein lysate $(25 \mu \mathrm{g})$ was resolved in $8 \%$ SDSPAGE and hybridized with antibodies to EGFR (A) and phosphotyrosine (B). -, No EGF treatment; +, the cells were treated with $50 \mathrm{ng} / \mathrm{ml}$ EGF for $30 \mathrm{~min}$. The experiment was repeated for at least four times with similar results. A representative result is shown. MW, molecular weight.

region of topoII (5'-AGGCTGCAATGGTGACACTT-3') (nucleotides: 131-150) cDNA were designed. Sense oligonucleotides with a sequence complementary to the antisense one were used as the control. All bases were phosphorothioated to increase the stabilization of the oligonucleotides (Biobasic Inc., Canada). The cells were washed once with serum-free DMEM medium before transfection with the oligonucleotides. The oligonucleotides $(200 \mathrm{nM})$ were allowed to form a complex with oligofectamine (Invitrogen) in serum-free DMEM medium for $20 \mathrm{~min}$ at room temperature. Thereafter, the cells were incubated with the oligofectamine/oligonucleotides complex for $4 \mathrm{~h}$ at $37^{\circ} \mathrm{C}$. After transfection, the cells were either lysed for Western blot analysis or incubated in a complete medium for drug treatment.

\section{Results}

The effect of long-term EGF treatment on growth factor response in A431 cells. Incubation with $50 \mathrm{ng} / \mathrm{ml} \mathrm{EGF}$ for 30 weeks altered the EGF response in A431 cells. As shown in Fig. 1, EGF suppressed the growth of AP cells but stimulated the growth of AC cells. There was, however, no apparent change in the EGF receptor protein level in cells after long-term EGF treatment as indicated by the results from Western blot analysis (Fig. 2A). In addition, the patterns for both basal and EGF induced protein tyrosine phosphorylation were not affected by the long-term EGF 

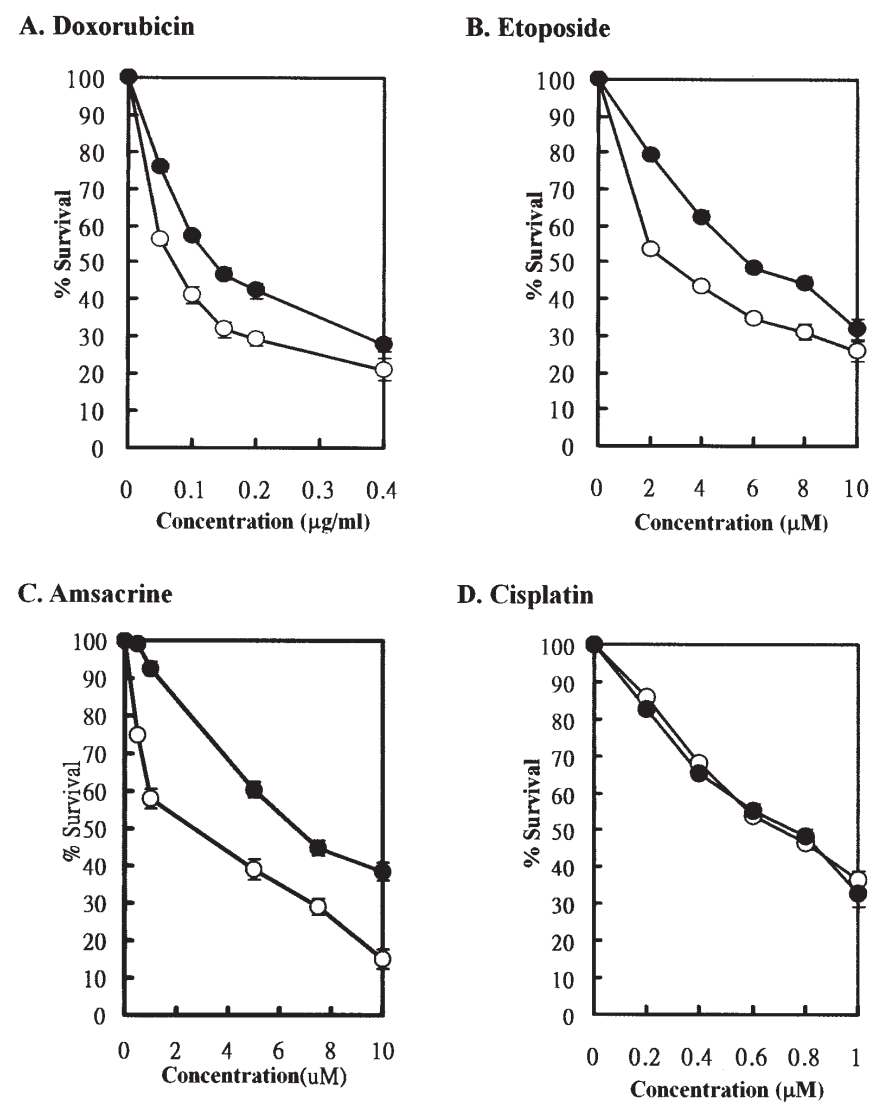

Figure 3. The sensitivity of A431 cells to (A) doxorubicin, (B) etoposide, (C) amsacrine and (D) cisplatin. Cells were grown for 2 days followed by $48 \mathrm{~h}$ of drug incubation. The cell survival was measured by MTT assay. Each data point is the average of results from at least 4 separate experiments. Error bar, standard derivation. $-\mathrm{O}-$, AP cells; - $\bullet-$, AC cells.

treatment as the patterns are similar between AP and AC cells (Fig. 2B).

The effect of long-term EGF treatment on anti-cancer drug sensitivity. The influence of long-term exposure to EGF on the sensitivity of cells to anticancer drugs was studied by MTT assay. Compared to AP cells, AC cells appeared to be more resistant to classical DNA topoII inhibitors such as doxorubicin, etoposide and amsacrine, whereas the sensitivity to cisplatin was similar (Fig. 3A-D). The IC50 values for doxorubicin, etoposide and amsacrine were about 2-, 2.5- and 3 -fold higher respectively in the AC cells than those in the AP cells. Results supported the fact that long-term exposure to EGF may modify the sensitivity of cells to anticancer drugs.

The effect of long-term EGF treatment on topoII expression. In an attempt to investigate the underlying mechanisms for the drug resistance induced by long-term EGF treatment, the levels of several drug resistance-related proteins in AP and AC cells were compared. Compared to AP cells, a 5-fold reduction of basal topoII protein level was observed in AC cells while there was no apparent difference in the expressions of other proteins such as P-glycoprotein, MRP-1 and glutathione-S-transferase- $\pi$ (GST- $\pi$ ) (Fig. 4).

TopoII expression and drug resistance in A431 cells. The downregulation of the topoII level in cells is known to be

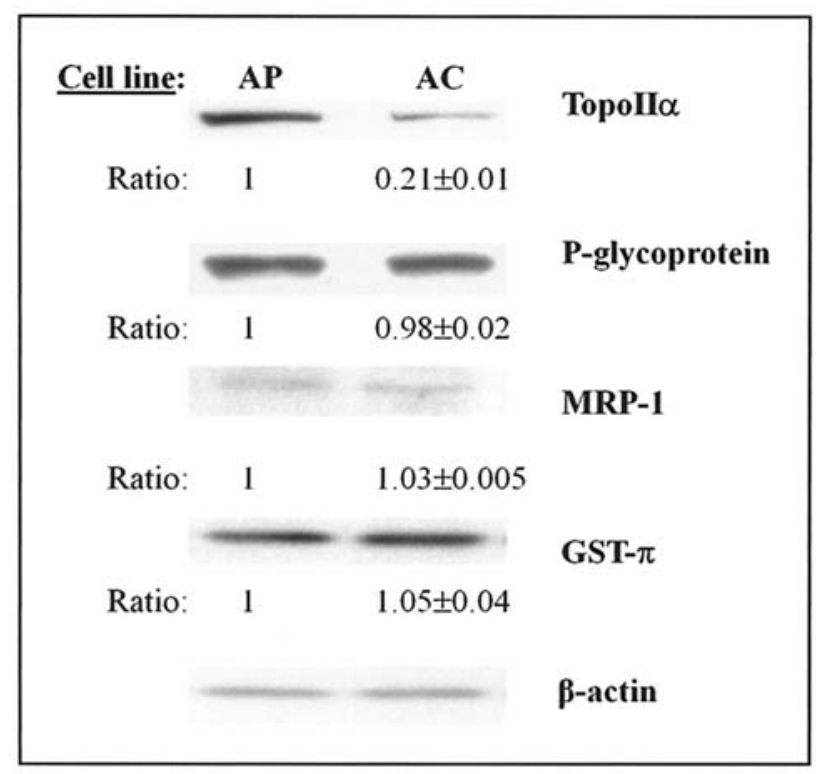

Figure 4. The protein levels of topoisomerase II $\alpha$ (TopoII), P-glycoprotein, MRP-1 and GST- $\pi$ in A431 cells as measured by Western blot analysis. Protein lysate $(25 \mu \mathrm{g})$ was resolved in $8 \%$ SDS-PAGE and was hybridized with the respective antibody. The value beneath the individual band represents the relative expression of the protein band. The expression of the protein band in the individual sample was first normalized by the expression of $\beta$ actin. The relative expression of each protein band (mean \pm SD of four sets of experiments) in AC cells was then calculated with respect to the expression in AP cells of which the expression is designated as 1 .

associated with resistance to topoII toxins, such as etoposide and amsacrine. To confirm if this is the case in A431 cells, the topoII level in the cells was suppressed by transfection with antisense topoII oligonucleotides and the sensitivity of the transfectants to doxorubicin, etoposide and amsacrine were examined by MTT assay. As shown in Fig. 5, topoII anti-sense oligonucleotides transfection suppressed the topoII protein level and increased the survival of the cells upon treatment with etoposide, doxorubicin and amsacrine in AP cells. The changes in topoII level and drug sensitivity were however not observed in AP cells transfected with sense topoII oligonucleotides (Fig. 5). In the case of the AC cells which already expressed a low level of topoII, even though the antisense oligonucleotides were found to knockdown, while the sense oligonucleotides had no effect on the topoII level, the transfection with either topoII sense or antisense oligonucleotides did not affect the drug sensitivity of the cells (Fig. 5). The results confirmed the important role of topoII in modifying drug resistance in A431 cells.

\section{Discussion}

Long-term EGF treatment decreased the cytotoxic effect of chemotherapeutic agents through the down-regulation of topoII in A431 cells. TopoII is an enzyme involves in the regulation of DNA topology by cutting both strands of DNA transiently thus relaxing the supercoiling in the molecule. Drugs targeting topoII induce irreversible DNA damage by binding covalently to the enzyme-DNA complex during the transition stage of the cleavage action. It is also known that depletion of topoII induces resistance to topoII toxins in cells 

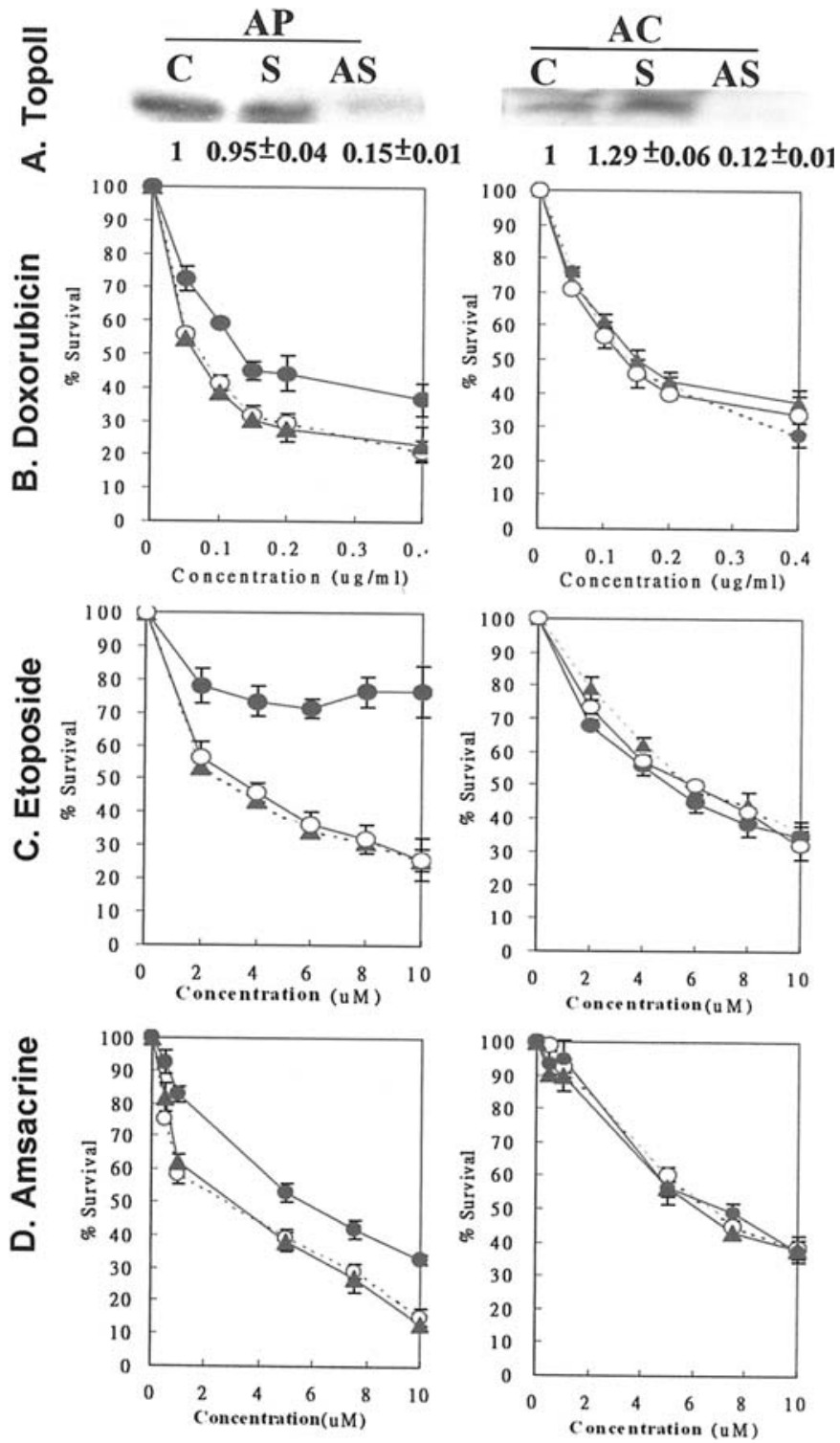

Figure 5. The effect of topoII oligonucleotide transfection on (A) topoII protein expression and the cytotoxic effects of (B) doxorubicin, (C) etoposide, and (D) amsacrine in AP cells (left panel) and AC cells (right panel). The cells were transfected with $200 \mathrm{nM}$ of oligonucleotides for $4 \mathrm{~h}$. Thereafter, the cells were lysed for Western blot analysis or treated with drugs for $48 \mathrm{~h}$ followed by the MTT assay. (A) Relative expression of each protein band (mean $\pm \mathrm{SD}$ of four sets of experiments) is shown below the individual band. The expression of the protein band in individual samples was first normalized by the expression of $\beta$-actin (not shown). The relative expression of each protein band in the cells was then calculated with respect to the cells with no transfection, of which the expression is designated as 1 . (B-D) -o-, cells with no oligonucleotide transfection; - - - , cells transfected with topoII sense oligonucleotides; - $\bullet-$, cells transfected with topoII antisense oligonucleotides. Each data point is the average of results from at least 4 separate experiments. Error bar, standard deviation.

(17-19). Long-term treatment of A431 cells with EGF resulted in the downregulation of topoII but not the levels of other drug resistance factors such as P-glycoprotein, MRP-1 and GST- $\pi$. Furthermore, the cells subjected to long-term EGF treatment were resistant to topoII toxin, such as doxorubicin, etoposide, amsacrine, but not to non-topoII toxin, e.g. cisplatin. The correlation between the changes in drug sensitivity and the cellular topoII level in cells after long-term EGF treatment suggests that the drug resistance detected in the AC cells is likely to be associated with topoII down-regulation in cells. The hypothesis was confirmed further as antisense topoII oligonucleotides were able to knockdown the topoII level and also drug sensitivity in A431 cells. Short-term exposure to EGF has been shown to modify the sensitivity of cells to topoII-targeting drugs through rapid and transient inhibition of topoII activity $(10,20)$. The results from the present study indicate that a similar enhancement effect on topoII toxin sensitivity can also be seen in cells with long-term EGF treatment. One should however be aware that the reductions in the topoII level and topoII toxin sensitivity after long-term EGF treatment are not a transient effect as both were still detected in the AC cells after the cells were cultured in a medium without EGF supplement for more than 20 weeks (results not shown).

Long-term EGF treatment altered the EGF growth response pattern but not the EGFR level in A431 cells. EGF suppressed the growth of the AP cells but not that of the AC cells. The EGFR level in AP and AC cells, however, was similar. Continuous exposure to inhibitors of various signaling molecules, e.g. genistein, suramin induced the resistance to the respective inhibitor. In addition, treatment with EGFRblocking antibodies (such as C225) in vivo induced the resistance to the therapeutic antibodies while the treatment did not alter the EGFR level in cells (12-15). Therefore, the results for the loss of the EGF growth suppression effect and no change in EGFR levels in A431 cells after long-term EGF treatment are comparable to those reported in other cell systems, in particular, those treated with EGFR-blocking antibodies.

In summary, the results from the present study suggest that long-term EGF treatment may down-regulate topoII expression and thus may have a major bearing on the chemotherapeutic response of cancer cells.

\section{Acknowledgements}

This study was supported by an Earmarked Grant (CUHK 4024/01M) from Research Grants Council, Hong Kong, and a Direct Grant from The Chinese University of Hong Kong, Hong Kong.

\section{References:}

1. Ling YH, Donato NJ and Perez-Soler R: Sensitivity to topoisomerase I inhibitors and cisplatin is associated with epidermal growth factor expression in human cervical squamous carcinoma ME180 sublines. Cancer Chemother Pharmacol 47: 473-480, 2001.

2. Lui VW and Grandis JR: EGFR-mediated cell cycle regulation. Anticancer Res 22: 1-11, 2002.

3. Herbst RS: Review of epidermal growth factor receptor biology. Int J Radiat Oncol Biol Phys 59: 21-26, 2004.

4. Lockhart $\mathrm{C}$ and Berlin JD: The epidermal growth factor receptor as a target for colorectal cancer therapy. Semin Oncol 32: 52-60, 2005.

5. Singh $A B$ and Harris RC: Autocrine, paracrine and juxtacrine signaling by EGFR ligands. Cell Signal (In press).

6. Kasid U: Raf-1 protein kinase, signal transduction, and targeted intervention of radiation response. Exp Biol Med 226: 624-625, 2001.

7. Kim R, Tanabe K, Uchida Y, Osaki A and Toge T: The role of HER-2 oncoprotein in drug sensitivity in breast cancer. Oncol Rep 9: 3-9, 2002. 
8. Tang WY, Chau SP, Tsang WP, Kong SK and Kwok TT: The role of Raf-1 in radiation resistance of human hepatocellular carcinoma Hep G2 cells. Oncol Rep 6: 1349-1354, 2004.

9. Kwok TT and Sutherland RM: Epidermal growth factor reduces resistance to doxorubicin. Int J Cancer 49: 73-76, 1991.

10. Allen GC, Lubas S, Wax MK and Devore RF: Epidermal growth factor regulates topoisomerase II activity and drug sensitivity in human KB cells. Otolaryngol Head Neck Surg 114: 785-792, 1996.

11. Cenni B, Aebi S, Nehme A and Christen RD: Epidermal growth factor enhances cisplatin-induced apoptosis by a caspase 3 dependent pathway. Cancer Chemother Pharmacol 47: 397-403, 2001.

12. Markovits J, Junqua S, Goldwasser F, Venuat AM, Luccioni C, Beaumatin J, Saucier JM, Bernheim A and Jacquemin-Sablon A: Genistein resistance in human leukemic CCRF-CEM cells: selection of a diploid cell line with reduced topoisomerase II beta isoform. Biochem Pharmacol 50: 177-186, 1995.

13. Lelievre S, Benchokroun Y and Larsen AK: Altered topoisomerase I and II activities in suramin-resistant lung fibrosarcoma cells. Mol Pharmacol 47: 898-906, 1995.

14. Viloria-Petit A, Cromber T, Jothy S, Hicklin D, Bohlen P, Schlaepppi JM, Rak J and Kerbel RS: Acquired resistance to the antitumour effect of epidermal growth factor-blocking antibodies in vivo: a role for altered tumour angiogenesis. Cancer Res 61: 5090-5101, 2001.
15. Ciardiello F, Bianco R, Caputo R, Damiano V, Troiani T, Melisi D, DeVita F, De Placido S, Bianco AR and Tortora G: Antitumour activity of ZD6474, a vascular endothelial growth factor receptor tyrosine kinase inhibitor, in human cancer cells with acquired resistance to antiepidermal growth factor receptor therapy. Clin Cancer Res 10: 784-793, 2004.

16. Fortune JM and Osheroff N: Topoisomerase II as a target for anticancer drugs: when enzyme stop being nice. Prog Nucleic Acid Res Mol Biol 64: 221-253, 2000.

17. Lage H, Helmbach H, Dietel M and Schadendort D: Modulation of DNA topoisomerase II activity and expression in melanoma cells with acquired drug resistance. Br J Cancer 82: 488-491, 2000.

18. Larsen AK, Escargucil AE and Skladanowski A: Catalytic topoisomerase II inhibitors in cancer therapy. Pharmacol Ther 99: 167-181, 2003.

19. Meresse P, Dechaux E, Monneret $C$ and Bertounesque E: Etoposide: discovery and medicinal chemistry. Curr Med Chem 11: 2443-2466, 2004

20. Harris LN, Yang L, Liotcheva V, Pauli S, Iglehart JD, Colvin OM and Hsieh TS: Induction of topoisomerase II activity after ErbB2 activation is associated with a differential response to breast cancer chemotherapy. Clin Cancer Res 7: 1497-1504, 2001 